\title{
Effect of Salacca Vinegar Var. Suwaru on Lipid Profile Diabetic Rats
}

\author{
Elok Zubaidah, Desta Yossy Ichromasari, Oty Kiki Mandasari \\ Department of Food Science and Technology, Agricultural Technology Faculty, Brawijaya University, Malang, \\ Indonesia \\ Email: elzoeba@yahoo.com, elok@ub.ac.id
}

Received 21 January 2014; revised 21 February 2014; accepted 28 February 2014

Copyright @ 2014 by authors and Scientific Research Publishing Inc.

This work is licensed under the Creative Commons Attribution International License (CC BY). http://creativecommons.org/licenses/by/4.0/

(c) (i) Open Access

\begin{abstract}
Diabetes mellitus is a group of metabolic diseases with hyperglycemia characteristic that occurs because of abnormalities of insulin secretion. And it becomes one of the major degenerative diseases in the world. The number of studies is conducted to produce hypoglycemic compound and hipolipidemia as an alternative to chemical drugs. One of natural product is salacca vinegar. The purpose of this research is to determine the effect of Salacca vinegar var. Suwaru impact on the diabetic lipid profile of male rats that induced by STZ (Streptozotocin). This reseacrh is conducted by Experimental Post Test Randomized Control Design. Under this research, involves 4 groups, spesifically normal group, diabetic group, diabetic + salacca vinegar $0.4 \mathrm{cc}$, diabetic + salacca vinegar $0.7 \mathrm{cc}$. The data were analyzed by ANOVA (Analysis of Variance). The means were assessed for differences through Least Significant Difference (LSD) 0.05. The results showed that Salacca vinegar var. Suwaru with a dose of $0.4 \mathrm{cc} / \mathrm{rat} / \mathrm{day}$ and $0.7 \mathrm{cc} / \mathrm{rat} / \mathrm{day}$ influence on lipid profile levels of diabetic rats which affect on decreasing LDL, Trygliceride, total cholesterol and increasing HDL serum plasma diabetic rats.
\end{abstract}

\section{Keywords}

Salacca Vinegar; Diabetic Rats; Dyslipidemia

\section{Introduction}

Diabetes mellitus is one of the major chronic diseases in all populations. In addition to hyperglycemia, the most common feature in diabetes is dyslipidemia, which is contributed to the development of atherosclerosis [1]. Diabetes mellitus is a disease that occurs when the body does not have the hormone insulin or cannot use insulin properly. Dyslipidemia is a metabolic abnormality of body fat. Glucose cannot be used as a source of energy so that the energy obtained from the breakdown of fat (lipolysis). Activity lipolysis (breakdown of fat) causes un- 
controlled high levels of free fatty acids, triglycerides (hipertrigliseridaemia) and cholesterol (hypercholesterolemia) can trigger the risk of cardiovascular complications such as atherosclerosis [2]. Diabetes mellitus to be a health concern is due to the prevalence of patients increased dramatically over the last two decades. The number of studies is conducted to produce hypoglycemic compound and hipolipidemia as an alternative to chemical drugs.

Vinegar is a product resulting from the fermentation of fruits that contain high levels of sugar such as apples, grapes etc. The main component in salacca vinegar is acetic acid with a concentration of $3 \%-5 \%$. Vinegars contain other components such as vitamins, mineral salts, amino acids, polyphenolic compounds and organic acids [3]. Supplement apple vinegar on diabetic rats showed positive results that can lower glucose levels and lipid content in the blood, polyphenols in apple have been shown to lower LDL levels in healthy individuals [1]. Acetic acid can lower triglycerides in rat fed a high-cholesterol diet [4], and the provision of vinegar can lower the glycemic index so as to increase HDL and decrease LDL [5] [6].

Salacca fruits have a higher content of antioxidants than apples [7]. In addition, salacca fruit is also high in polyphenols thus might have a positive effect to control of plasma lipids in rat fed a high-cholesterol diet [8]. Salacca vinegar is vinegar made form salacca fruits, which in some cases have a higher functional ability than apple vinegar [7]. Salacca vinegar may have the ability to control lipids in diabetic subjects. However, in-depth research on the subject has not been done. Therefore it is necessary to study the effect of salacca vinegar on lipid profile in male Wistar rats were conditioned with Streptozotocin-induced diabetic (STZ).

\section{Materials and Methods}

\subsection{Materials}

White rats Rattus novergicus Wistar strain, 3 - 4 months old, 130 - 180 g body weight. Normal diet: comfeed PAR-S 67\%, Wheat flour 33\%. Salacca vinegar var. Swaru from laboratory of microbiology, dept of Food Science and Technogy, Brawijaya University, Standard feed rats, Streptozotocin (Sigma Chemical).

\subsection{Experimental Design}

The animals were randomly divided into four groups and each group consists of 5 rats, classified as: normal, diabetic control, diabetic rats treated with salacca vinegar 0.4 cc doses, diabetic rats treated with salacca vinegar 0.7 cc doses. Each group is separated in different cages. Before treatment rats adapted to the laboratory conditions for 1 week in order to adapt to the environment and given a normal diet (standard).

\subsection{Determination of Dose Treatment}

Dose of salacca vinegar salacca commonly used by humans is $3 \times 2$ tablespoons salacca vinegar 2 tablespoons salacca vinegar $=10 \mathrm{cc}$. Dosage administered to rat can be calculated by multiplying the dosage in humans with human-to-rat conversion factor is 0.0027 , so the dosage obtained for rats weighing $150 \mathrm{~g}$ as follows: $0.0027 \mathrm{cc} / \mathrm{g}$ $\mathrm{BB}$ rat $\times 150 \mathrm{~g}=0.4 \mathrm{cc} / \mathrm{rat} /$ days.

\subsection{Induction of Diabetes}

Treatment of diabetes is done by injecting streptozotocin (STZ) at a dose of $60 \mathrm{mg} / \mathrm{kg}$ intraperitoneally [1]. Blood glucose levels measured 3 days after STZ induction. State of diabetic rat is characterized by blood glucose levels $\geq 200 \mathrm{mg} / \mathrm{dl}[1]$.

\subsection{Measurement of Lipid Profile Levels}

Blood samples were collected from the tail after $12 \mathrm{~h}$ fasting (before treatment), after the treatment period (end of the 28 days), blood sampling performed on 28 days, collected from the heart. Levels of total cholesterol, HDL (high density lipoprotein) and LDL (Low Density Lipoprotein) analyzed by CHOD-PAP method whereas triglyceride levels were analyzed by GPO-PAP method [2].

\subsection{Data Analysis}

The data were analyzed by ANOVA (Analysis of Variance). The means were assessed for differences through 
Least Significant Difference (LSD).

\section{Results}

\subsection{Effect of Salacca Vinegar on Blood Glucose Rat}

Rat induced by Streptozotocin (STZ). STZ injections influence increase in blood glucose levels above the normal range ( $>200 \mathrm{mg} / \mathrm{dl})$. Glucose levels after injection of streptozotocin rats can be seen in Table 1 .

Injection of STZ in animals causing diabetes mellitus. Changes in blood glucose abnormalities are due to pancreatic $\beta$-cell function. STZ pancreatic $\beta$ cells enter through the glucose transporter (GLUT-2) and will cause alkylation of DNA so that the DNA is fragmented. Pancreatic $\beta$ cell damage influence decrease in insulin levels will affect abnormality in the body's fat metabolism. Deficiency or lack of insulin resulting in increased blood glucose levels.

\subsection{Lipid Profiles}

Glucose obtained from food cannot be utilized as an energy source by the body so that the body uses energy from other sources, one of them from fat. Insulin deficiency causes inhibition of lipogenesis and increased lipolysis resulting in the mobilization of fatty acids from adipose tissue. Increased mobilization of fatty acids raises barriers in the glycolytic pathway, fatty acid synthesis, and encourage beta oxidation in liver to acetyl co-A is abundant. High levels of acetyl co-A in the liver would increase pathways that use acetyl co-A, the ketogenesis pathway and fatty acid synthesis. The total serum cholesterol, LDL, triglyceride and HDL did not significant different (5\%), in diabetic rat before treatment, but after 28 days there was change depend on of the treatment.

\subsubsection{LDL Level (Low Density Lipoprotein)}

LDL is a lipoprotein that has a low specific gravity so if contained in high amounts will cause thickening of blood vessel walls. Thickening of the walls of blood vessels forces the heart to work harder blood flow throughout the body and in the long term can lead to heart failure (cardiovascular diseases). Effect of salacca vinegar on blood serum LDL content of rats in various treatments can be seen in Table 2.

Table 1. Concentration of fasting blood glucose before and after 28 days treatment with salacca vinegar in normal and diabetic rats.

\begin{tabular}{ccc}
\hline \multirow{2}{*}{ Treatment group } & \multicolumn{2}{c}{ Blood glucose level (mg/dl) } \\
\cline { 2 - 3 } Normal & Before treatment & After treatment \\
Diabetic & $106 \mathrm{a}$ & $100 \mathrm{a}$ \\
Diabetic + salacca vinegar 0.4 cc & $350 \mathrm{~b}$ & $466 \mathrm{c}$ \\
Diabetic + salacca vinegar 0.7 cc & $404 \mathrm{~b}$ & $262 \mathrm{~b}$ \\
\hline
\end{tabular}

The values followed by different letters indicate significantly different test results on LSD 5\%.

Table 2. Concentration of serum low density lipoprotein cholesterol (LDL) before and after 28 days treatment with salacca vinegar in normal and diabetic rats.

\begin{tabular}{ccc}
\hline \multirow{2}{*}{ Treatment group } & \multicolumn{2}{c}{ Serum LDL content (mg/dl) } \\
\cline { 2 - 3 } Normal & Before treatment & After treatment \\
Diabetic & 8.03 & $9.73 \mathrm{a}$ \\
Diabetic + salacca vinegar 0.4 cc & 9.46 & $25.53 \mathrm{~b}$ \\
Diabetic + salacca vinegar 0.7 cc & 17.13 & $14.73 \mathrm{a}$ \\
\hline
\end{tabular}

The values followed by different letters indicate significantly different test results on LSD 5\%. 


\subsubsection{Triglyceride}

Effect of salacca vinegar on blood serum triglyceride of rats in various treatments can be seen in Table 3 .

\subsubsection{HDL Level (High Density Lipoprotein)}

HDL (High Density Lipoprotein) has the opposite function of LDL as well as some type of low density lipoproteins in the serum. Functioning HDL transports cholesterol from tissues to the heart. The serum levels of HDL in rat are shown in Table 4.

\subsubsection{Total Cholesterol}

The serum levels of total cholesterol rat for all treatment can be seen in Table 5 .

\section{Discussion}

The group of diabetic rats increased LDL, triglyceride, cholesterol levels compared to normal rat (Tables 2, 3

Table 3. Concentration of serum triglyceride before and after 28 days treatment with salacca vinegar in normal and diabetic rats.

\begin{tabular}{ccc}
\hline \multirow{2}{*}{ Treatment group } & \multicolumn{2}{c}{ Serum triglyceride content (mg/dl) } \\
\cline { 2 - 3 } Normal & Before treatment & After treatment \\
Diabetic & 54.66 & $56 \mathrm{a}$ \\
Diabetic + salacca vinegar 0.4 cc & 59 & $90.66 \mathrm{~b}$ \\
Diabetic + salacca vinegar 0.7 cc & 56 & $69.66 \mathrm{a}$ \\
\hline
\end{tabular}

The values followed by different letters indicate significantly different test results on LSD 5\%.

Table 4. Concentration of serum high density lipoprotein cholesterol (HDL) before and after 28 days treatment with salacca vinegar in normal and diabetic rats.

\begin{tabular}{ccc}
\hline \multirow{2}{*}{ Treatment group } & \multicolumn{2}{c}{ Serum HDL content (mg/dl) } \\
\cline { 2 - 3 } & Before treatment & After treatment \\
\hline Normal & 48.33 & $49 \mathrm{~b}$ \\
Diabetic & 46.03 & $32 \mathrm{a}$ \\
Diabetic + salacca vinegar 0.4 cc & 47.1 & $46.33 \mathrm{~b}$ \\
Diabetic + salacca vinegar 0.7 cc & 43.5 & $36.3 \mathrm{ab}$ \\
\hline
\end{tabular}

The values followed by different letters indicate significantly different test results on LSD 5\%.

Table 5. Concentration of serum total cholesterol before and after 28 days treatment with salacca vinegar in normal and diabetic rats.

\begin{tabular}{ccc}
\hline \multirow{2}{*}{ Treatment group } & \multicolumn{2}{c}{ Serum cholesterol content (mg/dl) } \\
\cline { 2 - 3 } & Before treatment & After treatment \\
\hline Normal (P0) & 57.66 & $56.66 \mathrm{a}$ \\
Diabetic (P1) & 71.66 & $78.66 \mathrm{~b}$ \\
Diabetic + salacca vinegar 0.4 cc (P2) & 75.33 & $65 \mathrm{a}$ \\
Diabetic + salacca vinegar 0.7 cc (P3) & 67.66 & $61 \mathrm{a}$ \\
\hline
\end{tabular}

The values followed by different letters indicate significantly different test results on LSD 5\%. 
and 5). The diabetics have abnormal lipid metabolism due to insulin deficiency in the body as a result of STZ induced damage to pancreatic $\beta$ cells. Insulin can activate lipoprotein lipase, the enzyme lipoprotein solver. On the diabetic condition of the enzyme lipoprotein lipase activity decreased so that the levels of lipoproteins in the blood increases [3]. In the body of triglyceride-rich lipoproteins are catabolized to inhibit the production of VLDL by the liver. LDL is the end product of VLDL, VLDL production so if possible cannot be controlled will be an increase in LDL levels in the body.

The group of diabetes by administering 0.4 cc salacca vinegar and 0.7 cc seen a decrease in LDL, cholesterol, triglyceride, levels compared to diabetic rat. Acetic acid or SCFA (short chain fatty acids) are known to lower the glycemic index (GI) [9]. Consumption of acetic acid contained in salacca vinegar is not only to improve but also prolong satiety. The existence of this satiety response slows the body's metabolism so that it will increase the response or insulin sensitivity in turning glucose into muscle sugar. Presence of acetic acid consumption or diet lowers concentrations of HMG-CoA is a substrate of the liver in the synthesis of cholesterol. Vinegar can also increase fat oxidation and stimulation of fecal excretion of bile acids [4].

In addition to acetic acid, compounds that have an important role to decrease LDL levels are polyphenolic compounds that contained in the salacca vinegar (tannins). Polyphenols in apple have been shown to inhibit the increase in serum cholesterol levels in the blood, increase the activity of fat-burning enzymes in the liver, the enzyme acyl CoA oxidation, decrease the activity of fatty acid synthase [10]. Synthase polyphenols can inhibit the secretion of cholesterol and lipoproteins [11]. Antinutrisi substances such as tannins can reduce cholesterol levels in the body by binding to bile acids in the intestine and excreted through feces. Additionally tannins can inhibit the action of HMG-CoA reductase.

Triglycerides are a form of fat that is absorbed by the gut after a hydrolysis, that goes into the plasma in two forms, namely as chylomicrons (derived from intestinal absorption of fat after a meal) and as VLDL (very low density lipoprotein) which is formed by the liver with help of insulin [12]. High levels of triglycerides related with insulin resistance or deficiency results in abnormal lipid metabolism. In these conditions, the activity of fat mobilization and lipolysis in the body increases. Glycerol levels increased lipolysis results will stimulate triglyceride synthesis. Reserves of fat in adipose tissue must be removed and distributed to all tissues via the circulatory system so that, levels of total cholesterol and triglycerides in the body increases.

Acetic acid in the salacca vinegar is absorbed and taken then metabolized via acetyl co-A in the tricarboxylic acid cycle in the liver and peripheral tissues. On the other hand, when acetic acid is metabolized by acetyl co-A synthase, AMP produced simultaneously. Hepatocytes acetate activated AMP which activates protein kinase (AMPK), inhibitors of fatty acid and sterol synthesis, and also reported that acetic acid can lower serum triglycerides (TG) in rat fed a cholesterol diet steeper [4]. Another addition to acetic acid compounds which allegedly provided securities decreased levels of triglycerides are tannins [13].

Table 4 shows that the levels of HDL in diabetic rats are lower than the normal group, but higher than diabetic rat. Decreased of HDL is due to the relations with insulin deficiency. Insulin deficiency causes inhibition of lipoprotein lipase enzyme activity that serves to break lipoprotein [14]. Levels of HDL also negatively correlated with triglyceride levels. Decrease triglyceride levels by treatment group followed by an increase in HDL levels. Triglyceride transfer to the liver will be able to increase the amount of HDL, because the rest of the release of protein will increase the formation of HDL triglyceride [15]. There is an inverse correlation between LDL to HDL.

Increased levels of HDL also influenced by the effects of polyphenols contained in salacca vinegar. Polyphenols compound in apple can increase serum HDL in rat. Mechanism of action of tannins in lowering cholesterol is known through several ways such as by inhibiting the absorption of cholesterol or by increasing the excretion of cholesterol through feces [10].

Table 5 shows an increase in total cholesterol in the group of diabetic rats compared with normal rats. Its due to lipid metabolism as result of a deficiency or insulin resistance. Increased levels of total cholesterol influence by the treatment group diabetes + salacca vinegar 0.4 cc and 0.7 cc. This was due to an increase in total cholesterol is the sum of all existing lipoproteins.

Total cholesterol is a combination of lipoproteins, which consists of $60 \%-70 \%$ LDL, HDL $20 \%-30 \%$, and $10 \%-15 \%$ of VLDL [16]. Total cholesterol is a combination levels of triglycerides, HDL and LDL levels. So if there is an increase HDL levels can affect the total cholesterol in the body [17]. In the diabetic group found highest levels of total cholesterol compared to the normal group. This is presumably due to increased levels of triglycerides and LDL cholesterol in the body. 


\section{Conclusion}

Salacca vinegar var. Suwaru with a dose of $0.4 \mathrm{cc} / \mathrm{rat} / \mathrm{day}$ and $0.7 \mathrm{cc} / \mathrm{rat} / \mathrm{day}$ influence on lipid profile levels of diabetic rats which affect on decreasing LDL, Trygliceride, total cholesterol and increasing HDL serum plasma diabetic rats.

\section{References}

[1] Shishehbor, F., Mansoori, A., Sarkaki, A.R., Jalali, M.T. and Latifi, S.M. (2008) Apple Cider Salacca Vinegar Attenuates Lipid Profile in Normal and Diabetic Rats. Pakistan Journal of Biological Sciences, 11, 2634-2638. http://dx.doi.org/10.3923/pjbs.2008.2634.2638

[2] Hernawan, U., Sutarno, E. and Setyawan, A.D. (2004) Hypoglycemic and Hypolipidemic Activity Leaves Water Extract Lagerstroemia (Lagerstroemia speciosa [L.] Pers.) on Diabetic Rats. J. Biopharmaceutical, 2, 15-23.

[3] Johston, C.S. and Gass, C.A. (2006) Salacca Vinegar: Medicinal Uses and Antiglysemic Effect. Medscape General Medicine, 8, 61 .

[4] Fushimi, T., Suruga, K., Oshima, Y., Momoko, M., Tsukamoto, Y. and Goda, T. (2006) Dietary Acetic Acid Reduces Serum Cholesterol and Triacylglycerols in Rats Fed a Cholesterol-Rich Diet. British Journal of Nutrition, 95, 916-924. http://dx.doi.org/10.1079/BJN20061740

[5] Leeds, A.R. (2002) Glycemic Index and Heart Diseases. The American Journal of Clinical Nutrition, 76, 286-289.

[6] Slyper, A., Jurva, J., Pleuss, J., Hoffmann, R. and Gutterman, D. (2004) Influence of Glycemic Load on HDL Cholesterol in Youth. The American Journal of Clinical Nutrition, 81, 376-379.

[7] Zubaidah, E. and Kurniawan, Y. (2011) The Influence of the Type of Fruit (Salak and Apples) and Concentration Yeast Bread (Instant Dry Yeast) Antioxidant and Antibacterial Activity against Vinegar Salak (Salacca zalacca) and Vinegar Apples (Malus sylvestris). Journal of Agricultural Technology, 14.

[8] Leontowics, H., Leontowicz, M., Drzewiecki, J., Jastrzebski, Z., Haruenkit, R., Poovarodom, S., Park, Y.S., Jung, S.T., Kang, S.G., Trakhtenberg, S. and Gorinstein, S. (2007) Two Exotic Fruits Positively Affect Rat's Plasma Composition. Food Chemistry, 102, 192-200. http://dx.doi.org/10.1016/j.foodchem.2006.05.046

[9] Ostman, E., Granfeldt, E.Y., Persson, L. and Bjorok, I. (2005) Salacca Vinegar Supplementation Lowers Glucose and Insulin Responses and Increases Satiety after a Bread Meal in Healthy Subjects. European Journal of Clinical Nutrition, 59, 983-988. http://dx.doi.org/10.1038/sj.ejcn.1602197

[10] Nagasako-Akazome, Y., Kanda, T., Ohtake, Y., Shimasaki, H. and Kobayashi, T. (2007) Apple Polyphenols Influence Cholesterol Metabolism in Healthy Subjects with Relatively High Body Mass Index. Journal of Oleo Science, 56, 416-428. http://dx.doi.org/10.5650/jos.56.417

[11] Vidal, R., Hernandez-Vallejo, S. and Pauquai, T. (2005) Apple Procyanidins Decrease Cholesterol Esterification and Lipoprotein Secretion in Caco-2/TC7 Enterocytes. The Journal of Lipid Research, 46, 258-268. http://dx.doi.org/10.1194/jlr.M400209-JLR200

[12] Huda, M.S. (2006) Inside Cholesterol. http://www.kafka.web.id

[13] Faradilla, R.H.F. (2010) Profile and Lipid Peroxidation Rat Experiment after Giving Peanut Flour Tempe Komak (Lablab purpureus (L.) Sweet). Thesis, Bogor Agricultural University, Bogor.

[14] Howard, B.V. (1987) Lipoprotein Metabolism in Diabetes Mellitus. The Journal of Lipid Research, 28, 613-628.

[15] Mayes, P.A. (1997) Lipid Transport and Storage. In: Murry, R.K., Granner, D.K., Mayes, P.A. and Rodwell, V.W., Eds., A. Hartono (Translators), Harper's Biochemistry, 24th Edition, EGC Book Medical Publishers, Jakarta.

[16] Mahan, L.K. and Stump, S.E. (2004) Krause’s Food, Nutrition, \& Diet Therapy. 9th Edition, Elsevier, Amsterdam.

[17] Friedewald, W.T., Levy, R.I. and Frederickson, D.S. (1972) Estimation of the Concentration Low-Density Lipoprotein Cholesterol in Plasma without Use the Preparative Ultracentrifuge. Clinical Chemistry, 18, 499-502. 